\title{
Improvement of Doubly Q-Switching and Mode-Locking Performance with Nd-Doped Mixed Vanadate Crystals
}

\author{
Gang Zhang ${ }^{1 *}$, Shengzhi Zhao ${ }^{2}$, Bin Zhao ${ }^{3}$, YonggangWang ${ }^{4}$ \\ 1 College of Science, China University of Petroleum (East China), Qingdao 266555, China. \\ 2 School of Information Science and Engineering, Shandong University, Jinan, China. \\ ${ }^{3}$ College of Chemistry and Chemical Engineering, Fuzhou University, Fuzhou, China. \\ ${ }^{4}$ Xi'an Institute of Optics and Precision Mechanics, Chinese Academy of Sciences, Xi'an, China. \\ * Corresponding author. email: gang1019@126.com \\ Manuscript submitted November 24, 2014; accepted January 17, 2015. \\ doi: 10.17706/ijapm.2015.5.2.137-143
}

\begin{abstract}
By using both the single-walled carbon nanotube saturable absorber (SWCNT-SA) and an acousto-optic (AO) modulator, the stably doubly Q-switched and mode-locked (QML) lasers with Nd-doped mixed vanadate crystals have been realized. The doubly QML laser characteristics are measured. The doubly Q-switching and mode-locking performances are found to be greatly improved by use of $\mathrm{Nd}: \mathrm{Gd}_{0.3} \mathrm{Lu}_{0.33} \mathrm{Y}_{0.37} \mathrm{VO}_{4}$ incomparison to that achieved with $\mathrm{Nd}: \mathrm{YVO}_{4}, \mathrm{Nd}: \mathrm{LuVO}_{4}$, and $\mathrm{Nd}: \mathrm{Lu}_{0.2} \mathrm{Y}_{0.8} \mathrm{VO}_{4}$. The experiment results show that the $\mathrm{Nd}: \mathrm{Gd}_{0.3} \mathrm{Lu}_{0.33} \mathrm{Y}_{0.37} \mathrm{VO}_{4}$ crystal is more favorable than $\mathrm{Nd}: \mathrm{YVO}_{4}, \mathrm{Nd}: \mathrm{LuVO}_{4}$, and $\mathrm{Nd}: \mathrm{Lu}_{0.2} \mathrm{Y}_{0.8} \mathrm{VO}_{4}$ for doubly QML lasers with AO modulator and SWCNT-SA.
\end{abstract}

Key words: SWCNT-SA, doubly QML laser, mixed vanadate crystal.

\section{Introduction}

Simultaneously Q-switched and mode-locked (QML) lasers can generate the bursts of ps pulses with the higher peak power than that of CW mode-locked pulses. These kinds of lasers have found wide application in the visible display, the material-processing, nonlinear optics and so on. Diode-pumped passively QML lasers with saturable absorbers can supply this kind of pulses and have the advantages of simplicity, compactness, low cost and high efficiency. Novel saturable absorbers with easy fabrication technology and ultra-fast recovery times are needed for the QML lasers. Recently Single-walled carbon nanotube saturable absorbers (SWCNT-SAs) were used in solid-state lasers owing to their broad wavelength range from 1 to 2 $\mu \mathrm{m}$, simple and inexpensive fabrication process as well as high speed third-order optical nonlinearity [1]-[5]. The experimental results indicated that SWCNT-SA was excellent saturable absorber for mode-locked lasers. However, the solely passively QML laser with SWCNT-SA has the poor shot-to-shot stability and reproducibility as well as the low controllability for the pulse repetition rate, which can be improved by adding an active modulator into the laser cavity [6]-[9], known as the dual-loss modulations. In these lasers, the repetition rate of the Q-switched envelope is controlled by the active switch while the mode-locked pulses inside the Q-switched envelope depend on both the actively modulated loss and the saturable absorption. Thus the double QML lasers with the active acousto-optic (AO) modulator and SWCNT-SA are worth expecting.

Neodymium-doped vanadate crystals, because of large absorption coefficients and wide absorption bandwidths, have been widely used in diode-pumped cw solid-state lasers. Among of them, Nd:BaYF [10], 
$\mathrm{Nd}: \mathrm{YVO}_{4}$ [11], and $\mathrm{Nd}: \mathrm{GdVO}_{4}$ [12] have been used in diode-pumped solid-state lasers with SWCNT-SA. On the other hand, Too-large emission cross-section and the short fluorescence line widths of vanadate crystals, which limit their energy storage significantly, are serious drawbacks in Q-switching operation regime [13]. In order to improve the performance of lasers, the double-mixed $\mathrm{Nd}: \mathrm{Gd}_{x} \mathrm{Y}_{1-x} \mathrm{VO}_{4}$, $\mathrm{Nd}: \mathrm{Lu}_{x} \mathrm{Gd}_{1-x} \mathrm{VO}_{4}$, and $\mathrm{Nd}: \mathrm{Lu}_{x} \mathrm{Y}_{1-x} \mathrm{VO}_{4}$ crystals have been grown and extensively studied as gain media for all-solid state picoseconds pulse source [13]-[15]. Due to the reduction of the stimulated emission cross-section and the inhomogeneous broadening of the fluorescence line widths, larger pulse energy and shorter pulse width can be obtained in the double-mixed crystals lasers than those in single vanadate crystal lasers. Recently, a new triple-mixed vanadate crystal Nd: $\mathrm{Gd}_{0.3} \mathrm{Lu}_{0.33} \mathrm{Y}_{0.37} \mathrm{VO}_{4}$ has been fabricated [16]. The physical parameters of $\mathrm{Nd}: \mathrm{Gd}_{0.3} \mathrm{Lu}_{0.33} \mathrm{Y}_{0.37} \mathrm{VO}_{4}$ were shown in Table 1 in comparison to $\mathrm{Nd}: \mathrm{YVO}_{4}$, $\mathrm{Nd}: \mathrm{LuVO}_{4}$, and $\mathrm{Nd}: \mathrm{Lu}_{0.2} \mathrm{Y}_{0.8} \mathrm{VO}_{4}$. It can be seen that $\mathrm{Nd}: \mathrm{Gd}_{0.3} \mathrm{Lu}_{0.33} \mathrm{Y}_{0.37} \mathrm{VO}_{4}$ has smaller stimulated emission cross-section and broader fluorescence line width which is very importance for shorter pulse and higher peak power in pulsed laser systems. The QML laser involves two dynamic processes of Q-switching and mode-locking. However, the effects of the stimulated emission cross-section and the fluorescence line width on the simultaneously QML lasers haven't been reported as far as we known.

Table 1. Comparison between Nd:Gd $\mathrm{Gd}_{0.3} \mathrm{Lu}_{0.33} \mathrm{Y}_{0.37} \mathrm{VO}_{4}, \mathrm{Nd}: \mathrm{Lu}_{0.2} \mathrm{Y}_{0.8} \mathrm{VO}_{4}, \mathrm{Nd}: \mathrm{LuVO}_{4}$, and $\mathrm{Nd}: \mathrm{YVO}_{4}$

\begin{tabular}{lcclc}
\hline Crystals & $\begin{array}{l}\text { Fluorescence } \\
\text { line widths }(\mathrm{nm})\end{array}$ & $\begin{array}{l}\text { Fluorescence } \\
\text { lifetime }(\mu \mathrm{s})\end{array}$ & $\begin{array}{l}\text { Absorption } \\
\text { cross section }\left(\mathrm{cm}^{2}\right)\end{array}$ & $\begin{array}{l}\text { Emission } \\
\text { cross section }\left(\mathrm{cm}^{2}\right)\end{array}$ \\
\hline $\mathrm{Nd}: \mathrm{YVO}_{4}$ & 0.8 & 98 & $57 \times 10^{-20}$ & $250 \times 10^{-20}$ \\
$\mathrm{Nd}: \mathrm{LuVO}_{4}$ & 1.5 & 95 & $69 \times 10^{-20}$ & $146 \times 10^{-20}$ \\
$\mathrm{Nd}: \mathrm{Lu}_{0.2} \mathrm{Y}_{0.8 \mathrm{VO}}$ & 5.1 & 106 & $15 \times 10^{-20}$ & $76 \times 10^{-20}$ \\
$\mathrm{Nd}: \mathrm{Gd}_{0.3 \mathrm{Lu}} \mathrm{Lu}_{0.33} \mathrm{Y}_{0.37} \mathrm{VO}_{4}$ & 6.2 & 96 & $13 \times 10^{-20}$ & $73 \times 10^{-20}$ \\
\hline
\end{tabular}

In this paper, by using both the single-walled carbon nanotube saturable absorber (SWCNT-SA) and an acousto-optic (AO) modulator, the doubly Q-switched and mode-locked (QML) lasers with $\mathrm{Nd:YVO}$, $\mathrm{Nd}: \mathrm{LuVO}_{4}, \mathrm{Nd}: \mathrm{Lu}_{0.2} \mathrm{Y}_{0.8} \mathrm{VO}_{4}$ and $\mathrm{Nd}: \mathrm{Gd}_{0.3} \mathrm{Lu}_{0.33} \mathrm{Y}_{0.37} \mathrm{VO}_{4}$ crystals are realized under the same cavity parameters. The experiment results show that due to broader fluorescence line width of $\mathrm{Nd}: \mathrm{Gd}_{0.3} \mathrm{Lu}_{0.33} \mathrm{Y}_{0.37} \mathrm{VO}_{4}$, the doubly QML Nd:Gd $\mathrm{Gd}_{0.3} \mathrm{Lu}_{0.33} \mathrm{Y}_{0.37} \mathrm{VO}_{4}$ laser can generate shorter pulse width and higher peak power in comparison with the $\mathrm{Nd}: \mathrm{LuVO}_{4}, \mathrm{Nd}: \mathrm{YVO}_{4}$, or $\mathrm{Nd}: \mathrm{Lu}_{0.2} \mathrm{Y}_{0.8} \mathrm{VO}_{4}$ lasers.

\section{Experiment Setup}

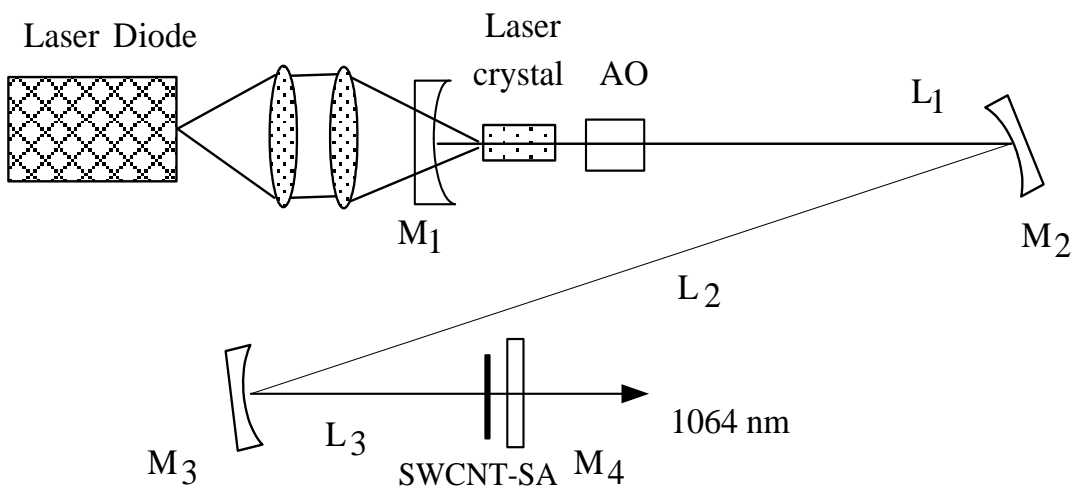

Fig. 1. Schematic diagram of the experimental laser setup. 
The laser cavity is shown in Fig. 1 A Z-type folded cavity is employed. Concave mirror $\mathrm{M}_{1}$ with radius of curvature of $200 \mathrm{~mm}$ transmits the pump radiation but reflects the generated laser pulses, functioning as the input mirror. Resonator mirror $\mathrm{M}_{2}$ and $\mathrm{M}_{3}$ are two concave mirrors with radii of curvature of 500 and $200 \mathrm{~mm}$, respectively. Both of them are HR coated at $1064 \mathrm{~nm}$. Flat mirror $\mathrm{M}_{4}$ acts as the output coupler (OC) and its reflectivity is $90 \%$. The distances between $M_{1}$ and $M_{2}\left(L_{1}\right), M_{2}$ and $M_{3}\left(L_{2}\right), M_{3}$ and $M_{4}\left(L_{3}\right)$ are 26,66 and $10 \mathrm{~cm}$, respectively.

The pump source employed is a fiber-coupled laser-diode array (Coherent, FAP system) which works at the maximum absorption wavelength $(808 \mathrm{~nm})$ of the $\mathrm{Nd}^{3+}$ ions. $\mathrm{Nd}: \mathrm{Gd}_{0.3} \mathrm{Lu}_{0.33} \mathrm{Y}_{0.37} \mathrm{VO}_{4}\left(0.5 \mathrm{at} . \% \mathrm{Nd}^{3+}, 3 \times 3 \times 5\right.$ $\left.\mathrm{mm}^{3}\right), \mathrm{Nd}: \mathrm{Lu}_{0.2} \mathrm{Y}_{0.8} \mathrm{VO}_{4}\left(0.5\right.$ at. $\left.\% \mathrm{Nd}^{3+}, 3 \times 3 \times 5 \mathrm{~mm}^{3}\right), \mathrm{Nd}: \mathrm{LuVO}_{4}\left(0.5\right.$ at. $\left.\% \mathrm{Nd}^{3+}, 3 \times 3 \times 2.5 \mathrm{~mm}^{3}\right)$ and $\mathrm{Nd}: \mathrm{YVO}_{4}$ (1.0at.\% $\mathrm{Nd}^{3+}, 3 \times 3 \times 5 \mathrm{~mm}^{3}$ ) are used as laser media in our experiment, respectively. They are all cut along the $a$-axis and the $3 \times 3 \mathrm{~mm}^{2}$ faces are polished and antireflection (AR) coated at $808 \mathrm{~nm}$ and $1064 \mathrm{~nm}$. To prevent thermally induced fracture and reduce the thermal effect, the gain medium is wrapped with a piece of thin indium foil and inserted into a slot in a water-cooled copper block, keeping at a temperature of $20^{\circ} \mathrm{C}$.

An acousto-optic (AO) modulator (The 26th Electronics Institute, Chinese Ministry of Information Industry) is used as active loss modulation. The effective length of AO modulator is $24 \mathrm{~mm}$ and both ends are AR coated at $1064 \mathrm{~nm}$. The SWCNT-SA (the quartz substrate coated with SWCNTs/PVA) is used as passive saturable absorption. The SWCNTs were grown by electric arc discharge technique, and the mean diameter is about $1.4 \mathrm{~nm}$.

The pulse temporal profile and repetition frequency are recorded by a Tektronix digital oscilloscope (500 MHz bandwidth and $2.5 \mathrm{Gs} / \mathrm{s}$ sampling rate, Tektronix Inc., USA) and a photo detector (New Focus, model 1623). Meanwhile the average output power is measured by a laser power meter (MAX 500AD, Coherent Inc., USA).

\section{Experimental Results and Discussions}

With an appropriate alignment of the laser cavity, stable QML laser emission was achieved. The doubly QML Nd:YVO $, \mathrm{Nd}^{2} \mathrm{LuVO}_{4}, \mathrm{Nd}: \mathrm{Lu}_{0.2} \mathrm{Y}_{0.8} \mathrm{VO}_{4}$, and $\mathrm{Nd}: \mathrm{Gd}_{0.3} \mathrm{Lu}_{0.33} \mathrm{Y}_{0.37} \mathrm{VO}_{4}$ lasers are all studied under the same cavity conditions. The modulation frequency of AO modulator is fixed as $10 \mathrm{kHz}$.

According to the repetition rate and the average output power, the pulse energy of the single Q-switched envelope versus the incident pump power for the four kinds of QML lasers can be calculated, which are shown with the scattered points in Fig. 2. From Fig. 2, we can see that the pulse energy of the Q-switched envelope almost increases with pump power. At the pump power of $7.09 \mathrm{~W}$, the obtained maximum pulse energy is $39.3 \mu \mathrm{J}$ for the double QMLNd:Gd $\mathrm{Gd}_{0.3} \mathrm{Lu}_{0.33} \mathrm{Y}_{0.37} \mathrm{VO}_{4}$ laser, which is higher than that obtained in the double QML Nd:YVO ${ }_{4}(28.11 \mu \mathrm{J}), \mathrm{Nd}: \mathrm{LuVO}_{4}(31.3 \mu \mathrm{J})$, and $\mathrm{Nd}: \mathrm{Lu}_{0.2} \mathrm{Y}_{0.8} \mathrm{VO}_{4}(36.1 \mu \mathrm{J})$ lasers. The pulse energy enhancement may result from the smaller stimulated emission cross-section and the broader fluorescence line width.

Fig. 3 shows the pulse width on incident pump power for four kinds of double QML lasers with the squares. From Fig. 3, it can be seen that the double QML Nd:Gd $\mathrm{Gd}_{0.3} \mathrm{Lu}_{0.33} \mathrm{Y}_{0.37} \mathrm{VO}_{4} \mathrm{l}$ aser can generate shorter pulse than that obtained in the double QML Nd:YVO ${ }_{4}, \mathrm{Nd}: \mathrm{LuVO}_{4}$, and $\mathrm{Nd}: \mathrm{Lu}_{0.2} \mathrm{Y}_{0.8} \mathrm{VO}_{4}$ lasers. Fig.4 shows the temporal shape of Q-switched pulse envelope for four kinds of QML lasers at the pump power of 7.09 W. The shortest pulse width of the dual-loss modulated QML Nd:YVO ${ }_{4}, \mathrm{Nd}: \mathrm{LuVO}_{4}, \mathrm{Nd}: \mathrm{Lu}_{0.15} \mathrm{Y}_{0.85} \mathrm{VO}_{4}$, and $\mathrm{Nd}: \mathrm{Gd}_{0.3} \mathrm{Lu}_{0.33} \mathrm{Y}_{0.37} \mathrm{VO}_{4}$ laser are $140,100,90$, and $80 \mathrm{~ns}$, respectively. In comparison with the double QML $\mathrm{Nd}: \mathrm{YVO}_{4}, \mathrm{Nd}: \mathrm{LuVO}_{4}$, and $\mathrm{Nd}: \mathrm{Lu}_{0.15} \mathrm{Y}_{0.85} \mathrm{VO}_{4}$ lasers, the double QML $\mathrm{Nd}: \mathrm{Gd}_{0.3} \mathrm{Lu}_{0.33} \mathrm{Y}_{0.37} \mathrm{VO}_{4}$ laser has compressed the pulse width by 43,20 and $11.1 \%$, respectively. Broader fluorescence line width of laser medium may result in shorter pulse width of Q-switched pulse envelope in QML laser.

According to the above results, the highest pulse energy and shortest pulse width can be obtained for the 
double QML Nd:Gd $\mathrm{Gd}_{0.3} \mathrm{Lu}_{0.33} \mathrm{Y}_{0.37} \mathrm{VO}_{4}$ laser. However for the QML laser, it is very difficult to directly measure the mode-locked pulse width in the Q-switched envelope even using an autocorrelation. So the mode-locked pulse width can be estimated by the formula [17].

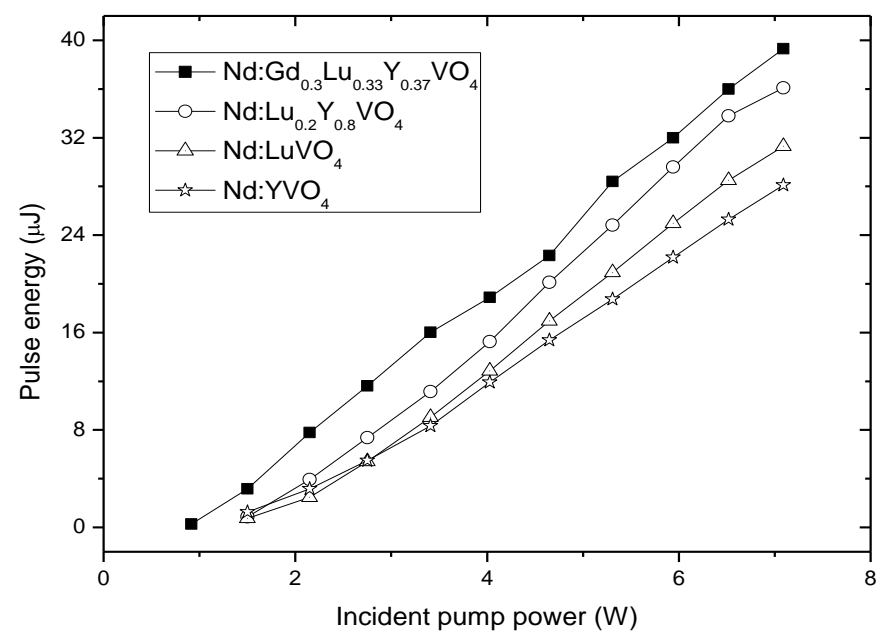

Fig. 2. Pulse energy of the Q-switched envelope versus the incident pump power.

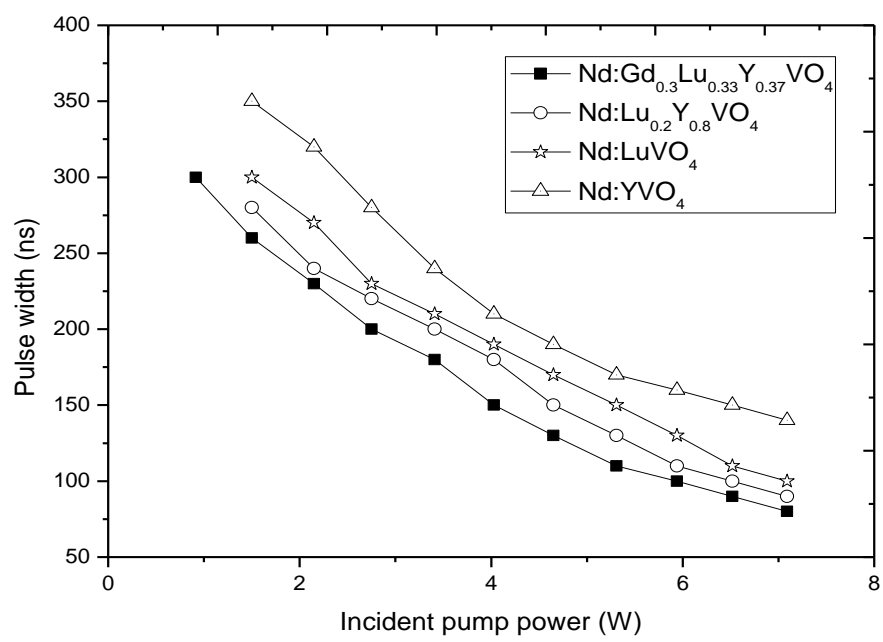

Fig. 3. Pulse width of Q-switched envelope versus incident pump power.

$$
t_{\text {real }}=\left(t_{\text {measure }}^{2}-t_{\text {probe }}^{2}-t_{\text {oscilloscope }}^{2}\right)^{1 / 2},
$$

Here, $t_{\text {real }}$ real of the pulse, $t_{\text {measure }}$ is the measured rise time, $t_{\text {probe }}$ is the rise time of the probe and $t_{\text {oscilloscope }}$ is the rise time of the oscilloscope. In our experiment, the average rise time of the mode-locked pulse in double QML Nd:Gd $\mathrm{Gd}_{0.3} \mathrm{Lu}_{0.33} \mathrm{Y}_{0.37} \mathrm{VO}_{4}$ laser is measured to be $1.4 \mathrm{~ns}$, which is shown in Fig. 5. The rise time of oscilloscope is $0.8 \mathrm{~ns}$, and the rise time of the probe is about $1 \mathrm{~ns}$. The real rise time of the mode-locked is about 565 ps. Then assuming the width of the pulse is approximately 1.25 times more than the rise time of the pulse, the estimated mode-locked pulse duration is about $706 \mathrm{ps}$. According to the pulse energy and the mode-locked pulse number inside the Q-switched envelope, the calculated maximum average mode-locked pulse peak power for double QML Nd:Gd0.3 $\mathrm{Lu}_{0.33} \mathrm{Y}_{0.37} \mathrm{VO}_{4}$ laser is $47.3 \mathrm{~kW}$ at the 
pump power of $7.09 \mathrm{~W}$.

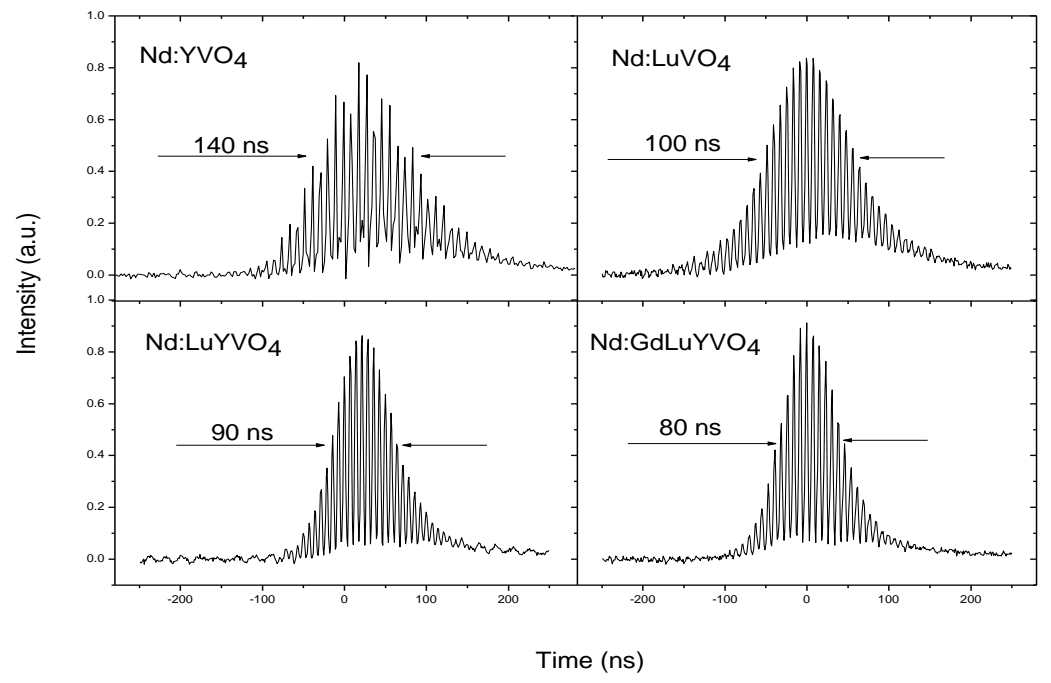

Fig. 4. Expanded temporal shape of a single QML pulse trains at 7.09 W pumping power for $\mathrm{Nd}: \mathrm{YVO}_{4}$, $\mathrm{Nd}: \mathrm{LuVO}_{4}, \mathrm{Nd}: \mathrm{Lu}_{0.15} \mathrm{Y}_{0.85} \mathrm{VO}_{4}$, and Nd:Gd $\mathrm{Gd}_{0.3} \mathrm{Lu}_{0.33} \mathrm{Y}_{0.37} \mathrm{VO}_{4}$.

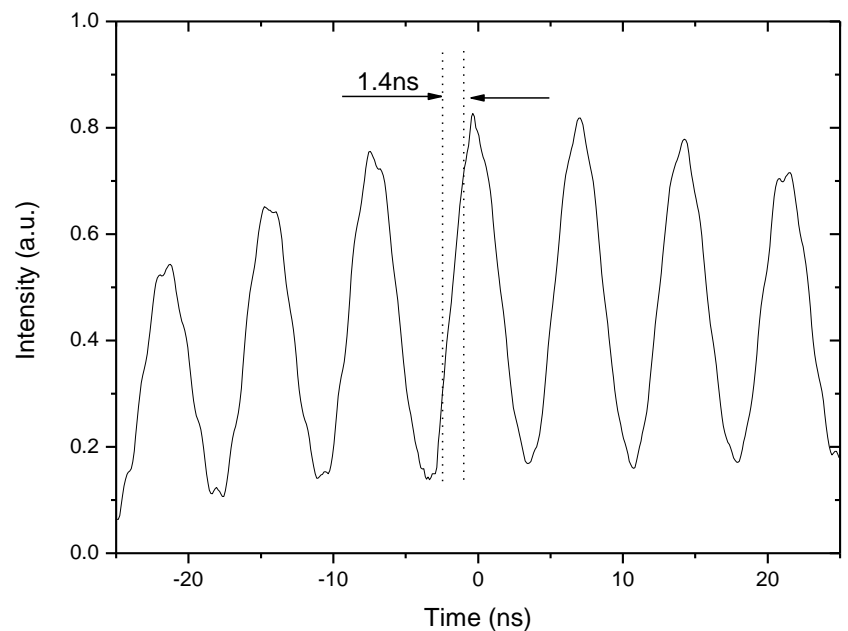

Fig. 5. Expanded oscilloscope traces of a train of mode-locked pulses at the pump power of $7.09 \mathrm{~W}$ for $\mathrm{Nd}: \mathrm{Gd}_{0.3} \mathrm{Lu}_{0.33} \mathrm{Y}_{0.37} \mathrm{VO}_{4}$.

\section{Conclusions}

In conclusion, by using both the single-walled carbon nanotube saturable absorber (SWCNT-SA) and

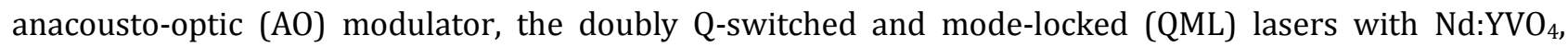
$\mathrm{Nd}: \mathrm{LuVO}_{4}, \mathrm{Nd}: \mathrm{Lu}_{0.2} \mathrm{Y}_{0.8} \mathrm{VO}_{4}$ and $\mathrm{Nd}: \mathrm{Gd}_{0.3} \mathrm{Lu}_{0.33} \mathrm{Y}_{0.37} \mathrm{VO}_{4}$ crystals have been realized. The experiment results show that the doubly QML Nd:Gd ${ }_{0.3} \mathrm{Lu}_{0.33} \mathrm{Y}_{0.37} \mathrm{VO}_{4}$ laser can generate shorter pulse width and higher peak power in comparison with the $\mathrm{Nd}: \mathrm{LuVO}_{4}, \mathrm{Nd}_{\mathrm{YVO}}$, or $\mathrm{Nd}: \mathrm{Lu}_{0.2} \mathrm{Y}_{0.8} \mathrm{VO}_{4}$ lasers. For the QML lasers, broader fluorescence line width of laser medium may result in shorter pulse width and higher peak power of Q-switched pulse envelope.

\section{Acknowledgment}


This work is partially supported by the National Science Foundation of China (61378022) and Fundamental Research Funds for the Central Universities (14CX02151A) and the Natural Science Foundation of Shandong Province(ZR2014FP008).

\section{References}

[1] Schibli, T. R., Minoshima, K., Kataura, H., Itoga, E., Minami, N., Kazaoui, S., et al. (2005). Ultrashort pulse-generation by saturable absorber mirrors based on polymer-embedded carbon nanotubes. Optics Express, 13, 8025-8031.

[2] Schmidt, A., Rivier, S., Steinmeyer, G., Yim, J. H., Cho, W. B., Lee, S., et al. (2008). Passive mode locking of Yb:KLuW using a single-walled carbon nanotube saturable absorber. Optics Letters, 33, 729-731.

[3] Zhang, L., Wang, Y. G., Yu, H. J., Sun, L., Guo, L., Hou, W., Tang, J., Lin, X. C., \& Li, J. M. (2011). 880 nm LD pumped passive Q_switched and mode_locked $\mathrm{Nd}_{2} \mathrm{YVO}_{4}$ Laser Using a single_walled carbon nanotube saturable absorber. Laser Physics, 21, 454-458.

[4] Garnov, S. V., Solokhin, S. A., Obraztsova, E. D., Lobach, A. S., Obraztsov, P. A., Chernov, A. I., et al. (2007). Passive mode-locking with carbon nanotube saturable absorber in $\mathrm{Nd}: \mathrm{GdVO}_{4}$ and $\mathrm{Nd}: \mathrm{Y}_{0.9} \mathrm{Gd}_{0.1} \mathrm{VO}_{4}$ lasers operating at $1.34 \mu \mathrm{m}$. Laser Physics, 4, 648-651.

[5] Liu, Y., Wang, Y., \& Liu, C. (2008). High power ultrafast Nd:YVO 4 laser mode locked by single wall carbon nanotube absorber. Applied Physics B, 104, 835-838.

[6] Zhao, S., Li, G., Li, D., Yang, K., Li, Y., Li, M., et al. (2010). Dual-loss-modulated Q-switched and mode-locked Nd:GdVO 4 laser with $\mathrm{AO}$ and $\mathrm{Cr}^{4+}: \mathrm{YAG}$ saturable absorber. Laser Physics Letters, 7, 29-33.

[7] Datta, P. K., Mukhopadhyay, S., \& Das, S. K. (2004). Enhancement of stability and efficiency of a nonlinear mirror mode-locked Nd:YVO ${ }_{4}$ oscillator by an active Q-switch. Optics Express, 12, 4041-4046.

[8] Theobald, C., Weitz, M., Knappe, R., Wallenstein, R., \& L'Huillier, J. A. (2008). Stable Q-switch mode-locking of $\mathrm{Nd}: \mathrm{YVO}_{4}$ lasers with a semiconductor saturable absorber. Applied Physics B, 92, 1-3.

[9] Li, T., Zhao, S. Z., Zhuo, Z., Yang, K. J., Li, G. Q., \& Li, D. C. (2010). Dual-loss-modulated Q-switched and mode-locked $\mathrm{YVO}_{4} / \mathrm{Nd}: \mathrm{YVO}_{4} / \mathrm{KTP}$ green laser with EO and $\mathrm{Cr}^{4+}: \mathrm{YAG}$ saturable absorber. Optics Express, $18,10315-10322$.

[10] Antonio, A., Luca, C., Federico, P., Giancarlo, R., Alessandra, T., Mauro, T., et al. (2010). Diode-pumped $\mathrm{Nd}: \mathrm{BaY}_{2} \mathrm{~F}_{8}$ picosecond laser mode-locked with carbon nanotubesaturable absorbers. Journal of the Optical Society of America B, 27, 2445-2448.

[11] Pan, S. D., Wang, Y. G., \& Tang, J. (2011). Diode-pumped passively Q-switched and Q-switch mode-locked $\mathrm{Nd}: \mathrm{YVO}_{4}$ laser using single-wall carbon nanotube based saturable absorber. Laser Physics, 21, 867-872.

[12] Chen, H.-R., Wang, Y.-G., Tsai, C.-Y., Lin, K.-H., Chang, T.-Y., Tang, J., \& Hsieh, W.-F. (2011). High-power, passively mode-locked $\mathrm{Nd}: \mathrm{GdVO}_{4}$ laser using single-walled carbon nanotubes as saturable absorber. Optics Letters, 36, 1284-1286.

[13] Liu, J. H., Wang, Z. P., Meng, X. L., Shao, Z. S., Ozygus, B., Ding, A., \& Weber, H. (2003). Improvement of passive Q-switching performance reached with a new $\mathrm{Nd}$-doped mixed vanadate crystal $\mathrm{Nd}: \mathrm{Gd}_{0.64} \mathrm{Y}_{0.36} \mathrm{VO}_{4}$. Optics Letters, 28, 2330-2332.

[14] Zhuo, Z., Li, S. G., Li, T., Shan, C. X., Jiang, J. M., Zhao, B., et al. (2010). Diode-end-pumped passively Q-switched Nd: $\mathrm{Y}_{0.8} \mathrm{Lu}_{0.2} \mathrm{VO}_{4}$ laser with $\mathrm{Cr}^{4+}: \mathrm{YAG}$ crystal. Optics Communications, 283, 1886-1888.

[15] Yu, H., Zhang, H., Wang, Z., Wang, J., Yu, Y., Tang, D., Xie, G., Luo, H., \& Jiang, M. (2009). Passive mode-locking performance with a mixed $\mathrm{Nd}: \mathrm{Lu}_{0.5} \mathrm{Gd}_{0.5} \mathrm{VO}_{4}$ crystal. Optics Express, 17, 3264-3269.

[16] Cheng, K., Zhao, S. Z., Yang, K. J., Li, G. Q., Li, D. C., Zhang, G., Zhao, B., \& Wang, Y. G. (2011). Diode-pumped passively Q-switched $\mathrm{Nd}: \mathrm{Lu}_{0.33} \mathrm{Y}_{0.37} \mathrm{Gd}_{0.3} \mathrm{VO}_{4}$ laser using a single-walled carbon nanotube saturable absorber. Laser Physics Letters, 8, 418-422. 
[17] Yang, K., Zhao, S., Li, G., Li, M., Li, D., Wang, J., \& An, J., (2007). Diode-pumped passively Q-switched mode-locked c-cut Nd:GdVO 4 laser with a GaAs coupler. Optical Materials, 29, 1153-1158.

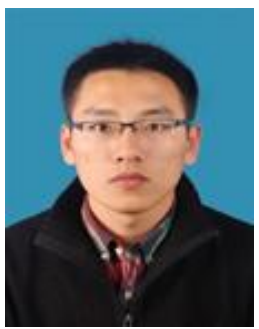

Gang Zhang received the B.Eng. degree in optoelectronics in 2006, and the Ph.D. degree in optical engineering in 2011, from the Shandong University, Jinan, China.

He is currently a lecturer at the College of Science, China University of Petroleum, East China. His research interests include laser technology, optoelectronics, and nonlinear optics.

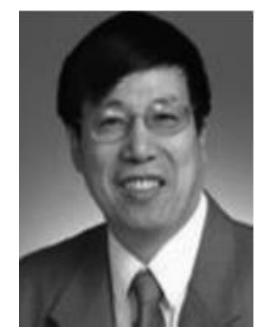

Shengzhi Zhao received the B.Sc. degree in lasers from the Shandong University, Jinan, China, in 1982, and the M.Sc. degree in optics from the Institute of Physics, Academia Sinica, Beijing, China, in 1986.

He is currently a professor at the School of Information Science and Engineering, Shandong University. His research interests include lasers, laser technology, laser applications, and nonlinear optics.

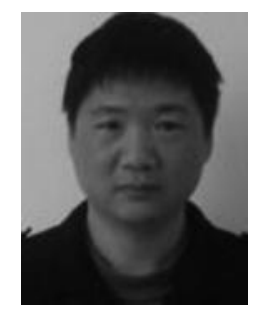

Bin Zhao received the B.Sc. degree in physical chemistry, in 1994, from the Wuhan University of Technology, Wuhan, China, and the M.Sc. degree in physical chemistry, in 2004, from the Fuzhou University, Fuzhou, China.

He is currently a lecturer at the College of Chemistry and Chemical Engineering, Fuzhou University. His research interests include laser technology and laser crystal materials.

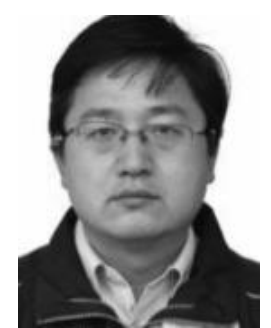

Yonggang Wang received the B.Sc. degree in physics, in 1997, from Xinjiang University, Urumqi, China, the M.Sc. degree in optics, in 2000, from the Shandong Normal University, Jinan, China, and the Ph.D. degree in optical engineering, in 2004, from the Institute of Semiconductors, Chinese Academy of Science, Beijing, China.

$\mathrm{He}$ is currently a research fellow at the Xi'an Institute of Optics and Precision Mechanics, Chinese Academy of Science. His research interests include laser technology, laser physics, laser application, and saturable absorption materials. 